\title{
Real-time Electron Microscopy of Protein Nanodiscs in Graphene Liquid Cells
}

\author{
$\underline{\text { John W. Smith }}{ }^{1}$, Lauren N. Carnevale ${ }^{2}$, Aditi Das ${ }^{2,3}$, and Qian Chen ${ }^{1,4 *}$
}

1. Department of Materials Science and Engineering, University of Illinois, Urbana, IL 61801, United States

2. Department of Biochemistry, University of Illinois, Urbana, IL 61801, United States

3. Department of Comparative Biosciences and College of Veterinary Medicine, University of Illinois, Urbana, IL 61801, United States

4. Department of Chemistry, Beckman Institute for Advanced Science and Technology, and Materials Research Laboratory, University of Illinois, Urbana, IL 61801, United States

*Corresponding author: qchen20@illinois.edu

Recent developments in the field of liquid-phase transmission electron microscopy (LP-TEM) are opening up new ways to investigate nanoscale dynamics at the single-particle [1] and even single-molecule level [2]. As such, there has been growing motivation to develop LP-TEM not just as a tool for the investigation of inorganic materials, but also for soft materials like polymers and biomolecules, whose complex nanoscale dynamics are intricately tied to their functionality. However, inherently low contrast, electron beam sensitivity, and other traits of soft materials have all served as technical obstacles to achieving this goal. Consequently, many LP-TEM investigations of soft materials have only been able to achieve a few snapshots of organic specimens, or low-resolution imaging that amounts to positional tracking, which can already be achieved with other techniques. Here, using a combination of graphene-based liquid cells, low dose imaging, and advanced image processing, we demonstrate that LP-TEM can be used to investigate the motion, rotation, and shape fluctuations of protein-based structures at the single-particle level.

In particular, we are interested in using LP-TEM as a tool to study large biomolecular complexes known as nanodiscs [3]. Over the past decade, nanodiscs were developed as a technology to investigate membrane proteins in a cell-free, single-particle format while preserving their native lipid environment. In this spirit, nanodiscs consist of a small patch of lipid bilayer containing one or more membrane proteins and surrounded by a "scaffold protein" that shields the hydrophobic regions of the lipid bilayer and makes the entire structure stable in solution. Nanodisc technology has previously been used to investigate membrane protein structure and dynamics using techniques such as small-angle X-ray scattering (SAXS) and nuclear magnetic resonance spectroscopy, as well as membrane protein catalytic activity in controlled lipid environments [4,5]. However, these methods are only capable of probing the ensemble biochemical and biophysical behavior of nanodiscs, whereas a method to study individual nanodisc dynamics would provide a platform to investigate phenomena ranging from protein-protein interactions to the conformational fluctuations of single membrane proteins in liquid.

Motivated by the potential of LP-TEM in this regard, we turned to graphene-based liquid cells, which offer the combined benefits of ultra-low background contrast and improved protection of beam-sensitive specimens [6], as a way to study these biomolecules. Using low-dose rate imaging and advanced image processing, we were able to image the rotation and diffusion of purified nanodiscs in their native buffer, across time spans on the order of a few minutes before degradation of the sample or liquid pocket. Figure 1 shows representative snapshots of the nanodisc projection over time. The dimensions of the nanodisc, including its major and minor axis dimensions and aspect ratio, are highly consistent with micrographs of negatively stained nanodiscs taken from the same sample and SAXS and size exclusion chromatography 
analysis. To further show that the observed particles correspond to nanodiscs, we prepared nanodiscs of different dimensions and aspect ratios and examined them in graphene liquid cells. In doing so, we saw a concomitant change in the nanodisc dimensions in the liquid cell, highlighting that graphene cell LP-TEM is a viable and promising platform on which to investigate the nanoscale dynamics of a wide variety of nanodiscs, and potentially other large biomolecules as well [7].

References:

[1] B Luo et al., Acc. Chem. Res. 50 (2017), p. 1125.

[2] KH Nagamanasa, H Wang, and S Granick, Adv. Mater. 29 (2017), 1703555.

[3] IG Denisov et al., J. Am. Chem. Soc. 126 (2004), p. 3477.

[4] IG Denisov and SG Sligar, Chem. Rev. 117 (2017), p. 4669.

[5] LN Carnevale et al., Biochemistry 57 (2018), p. 6489.

[6] H Cho et al., Nano Lett. 17 (2017), p. 414.

[7] The authors acknowledge funding from the Air Force Office of Scientific Research Young Investigator Program (AFOSR-YIP project FA9550-17-1-0296).
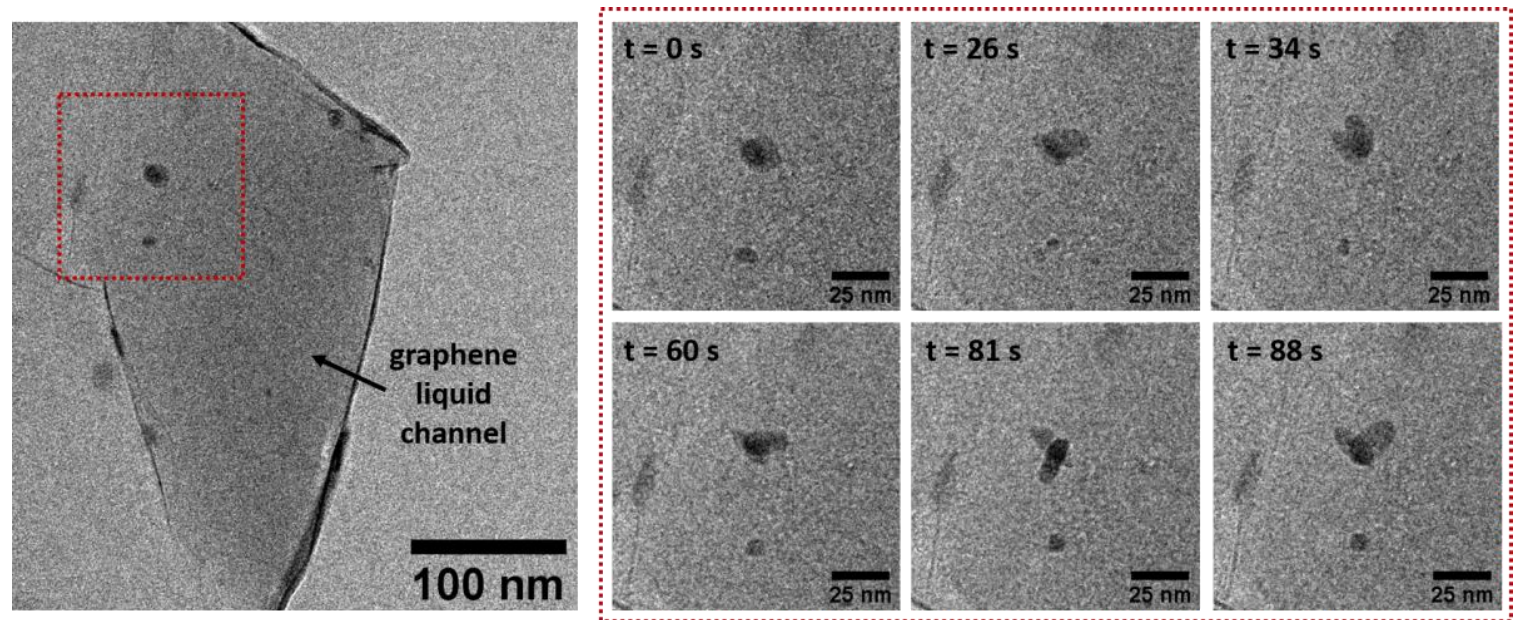

Figure 1: Snapshots of the rotational diffusion of a nanodisc encapsulated in its native buffer in a graphene liquid channel. The left-hand panel shows a zoomed out view of the complete channel, and the right-hand panel highlights the dynamic behavior of the nanodiscs over a sustained amount of time. 